Asim Zafar, Hadi Rezazadeh, and Khalid K. Ali*

\title{
On finite series solutions of conformable time-fractional Cahn-Allen equation
}

https://doi.org/10.1515/nleng-2020-0008

Received Jul 8, 2018; accepted Dec 28, 2019.

Abstract: The aim of this article is to derive new exact solutions of conformable time-fractional Cahn-Allen equation. We have achieved this aim by hyperbolic function and expa function methods with the aid of symbolic computation using Mathematica. This idea seems to be very easy to employ with reliable results. The time fractional Cahn-Allen equation is reduced to respective nonlinear ordinary differential equation of fractional order. Also, we have depicted graphically the constructed solutions.

Keywords: time-fractional Cahn-Allen equation; conformable derivatives; $\exp _{a}$ function method; hyperbolic function method; finite series solutions

\section{Introduction}

Fractional differential equation may be considered as the missing part of the classical differential equations. In recent years, many authors have studied the nonlinear fractional differential equations for example see [1-8] because these equations express many complex nonlinear physical phenomena and dynamic forms in physics. Several definitions of fractional derivative have been presented to the literature, amongst are Atangana Baleanu operator, CaputoFabrizio and conformable derivative.

In this research, we apply two methods on conformable time-fractional Cahn-Allen equation to scrutinize the new explicit exact solutions [9-12] that may read as

$$
D_{t}^{y}-u_{x x}+u^{3}-u=0,0<y \leq 1
$$

\footnotetext{
Asim Zafar, Department of Mathematics, CUI, Vehari Campus, Mailsi Road, Off Multan Road, Ada Peer Murad, Vehari, Punjab, Pakistan Hadi Rezazadeh, Faculty of Engineering Technology, Amol University of Special Modern Technologies, Amol, Iran

*Corresponding Author: Khalid K. Ali, Mathematics Department, Faculty of Science, Al-Azhar University, Nasr-City, Cairo, Egypt, E-mail: khalidkaram2012@yahoo.com
}

We employ the $\exp _{a}$ function approach [13-15] and the hyperbolic function approach [16-18] via traveling wave transformation with the conformable derivative.

The rest of the article is arranged as follows: In Section 2 some preliminaries and notations dealing with the fractional calculus theory are briefly described. Section 3 presents description of methods. The exact solutions of the nonlinear Cahn-Allen equation are constructed in Section 4. In Section 5 some graphical representation for solutions are showed. Section 6 presents the summary of the obtained results.

\section{Conformable derivative}

The latter part presents the results of the current study graphically.

We recall the conformable derivative with some of its properties [19].

Defination 1 Suppose $h: \mathbb{R}_{>0} \rightarrow \mathbb{R}$ be a function. Then, for all $t>0$,

$$
D_{t}^{\alpha}(p(t))=\lim _{\varepsilon \rightarrow 0} \frac{p\left(t+\varepsilon t^{1-\alpha}\right)-p(t)}{\varepsilon},
$$

is known as $\alpha, 0<\alpha \leq 1$ order conformable fractional derivative of $p$. The followings are some useful properties: $D_{t}^{\alpha}(a p+b g)=a D_{t}^{\alpha}(p)+b D_{t}^{\alpha}(g)$, for all $a, b \in \mathbb{R}$ $D_{t}^{\alpha}(p g)=p D_{t}^{\alpha}(g)+g D_{t}^{\alpha}(p)$

Let $p: \mathbb{R}_{>0} \rightarrow \mathbb{R}$ be an $\alpha$-differentiable function, $g$ be a differentiable function defined in the range of $p$.

$$
D_{t}^{\alpha}(p \circ g(t))=t^{1-\alpha} g^{\prime}(t) p^{\prime}(g(t)) .
$$

On the top of that, the following rules hold.

$D_{t}^{\alpha}\left(t^{h}\right)=h t^{h-\alpha}$, for all $h \in \mathbb{R}$

$D_{t}^{\alpha}(\delta)=0$, where $\delta$ is constant.

$D_{t}^{\alpha}(p / g)=\frac{g D_{t}^{\alpha}(p)-p D_{t}^{\alpha}(g)}{g^{2}}$.

Conjointly, if $p$ is differentiable, then $D_{t}^{\alpha}(p(t))=t^{1-\alpha} \frac{d p(t)}{d t}$. 


\section{Description of methods}

The present subsection provides a brief explanation for two reliable techniques in engendering new exact solutions to nonlinear conformable time-fractional equation. For this purpose, suppose that we have a nonlinear conformable time FDE that can be presented in the form

$$
F\left(u, D_{t}^{y} u, D_{x} u, D_{t t}^{2 y} u, D_{x x} u, \ldots\right)=0 .
$$

The FDE (2) can be changed into the following nonlinear ODE of integer order

$$
\wp\left(U, U^{\prime}, U^{\prime \prime}, \ldots,\right)=0,
$$

with the use of following wave transformation

$$
u(x, t)=U(\eta), \eta=k_{1} x-k_{2} \frac{t^{y}}{y},
$$

where $\wp$ is a polynomial in $U(\eta)$ and its total derivatives with $^{\prime}=d / d \eta$ while $k_{1}$ and $k_{2}$ are nonzero arbitrary constants.

\subsection{The $\exp _{a}$ function approach}

Let us try to search a non-trivial solution for (3) in the following form $[13-15,20]$

$$
U(\eta)=\frac{A_{0}+A_{1} a^{\eta}+\ldots+A_{N} a^{N \eta}}{B_{0}+B_{1} a^{\eta}+\ldots+B_{N} a^{N \eta}},
$$

where $A_{i}$ and $B_{i}$, for $(0 \leq i \leq N)$, are found later and $N$ is a free positive constant.

Replacing (5) in the nonlinear (3), yields

$$
\wp\left(a^{\eta}\right)=q_{0}+q_{1} a^{\eta}+\ldots+q_{\tau} a^{\tau \eta}=0 .
$$

Setting $l_{i}(0 \leq i \leq \tau)$ in (5) to be zero, results give a set of nonlinear equations as follows:

$$
q_{i}=0, \quad i=0, \ldots, \tau
$$

by solving the generated set (7), we acquire non-trivial solutions of the nonlinear PDE (2).

\subsection{The hyperbolic function approach}

Let us try to search a non-trivial solution to (3) in the following form [16-18, 21]

$$
U(\eta)=A_{0}+\sum_{i=1}^{N} \sinh ^{i-1}(\rho)\left[B_{i} \sinh (\rho)+A_{i} \cosh (\rho)\right],
$$

where $\rho$ is some specific functions. By calculating the positive integer $N$, setting (8) in (3), and comparing coefficients, we will find a set of nonlinear equations whose solution, finally provides explicit exact solutions of (2). It is worth mentioning that using the separation of variables techniques on $\frac{d \rho}{d \eta}=\sinh (\rho)$, we find $\sinh (\rho)= \pm \operatorname{csch}(\eta)$, $\cosh (\rho)=-\operatorname{coth}(\eta)$ and $\sinh (\rho)= \pm \imath \operatorname{sech}(\eta), \cosh (\rho)=$ $-\tanh (\eta)$. Accordingly, the solution (7) can be rewritten as

$$
\left.U(\eta)=A_{0}+\sum_{i=1}^{N}( \pm \operatorname{csch})^{i-1}(\eta)\right)\left[ \pm B_{i} \operatorname{csch}(\eta)-A_{i} \operatorname{coth}(\eta)\right],
$$

and

$$
U(\eta)=A_{0}+\sum_{i=1}^{N}( \pm \imath \operatorname{sech})^{i-1}(\eta)\left[ \pm \imath B_{i} \operatorname{sech}(\eta)-A_{i} \tanh (\eta)\right] .
$$

Likewise, it is obvious that from $\frac{d w}{d \eta}=\cosh (\rho)$, we find $\sinh (\rho)=-\cot (\eta), \cosh (\rho)= \pm \csc (\eta)$ and $\sinh (\rho)=$ $\tan (\eta), \cosh (\rho)= \pm \sec (\eta)$. Accordingly, the solution (7) can be rewritten as

$$
U(\eta)=A_{0}+\sum_{i=1}^{N}(-\cot )^{i-1}(\eta)\left[-B_{i} \cot (\eta) \pm A_{i} \csc (\eta)\right],
$$

and

$$
U(\eta)=A_{0}+\sum_{i=1}^{N}\left(\tan ^{i-1}\right)(\eta)\left[B_{i} \tan (\eta) \pm A_{i} \sec (\eta)\right] .
$$

\section{Application to time-fractional Cahn-Allen equation}

Using the transformation (4) in (1), we get

$$
k_{2} U^{\prime}-k_{1}^{2} U^{\prime \prime}-U+U^{3}=0 .
$$

Through balancing the terms $U^{\prime \prime}$ and $U^{3}$, we select $N=1$, the nontrivial solution (5) reduces to:

$$
U(\eta)=\frac{a_{1} a^{\eta}+a_{0}}{b_{1} a^{\eta}+b_{0}}, \quad a \neq 1
$$

By setting the above solution in (9) and equating factors of each power of $a^{\eta}$ in the resulting equation, we reach a set of nonlinear algebraic equations.

$$
\begin{aligned}
& a_{0}^{3}-a_{0} b_{0}^{2}=0, \\
& a_{1}^{3}-a_{1} b_{1}^{2}=0, \\
& a_{0} b_{0} b_{1} k_{1}^{2} \log ^{2}(a)-a_{1} b_{0}^{2} k_{1}^{2} \log ^{2}(a)+a_{0} b_{0} b_{1} k_{2} \log (a) \\
& -a_{1} b_{0}^{2} k_{2} \log (a)-2 a_{0} b_{0} b_{1}-a_{1} b_{0}^{2}+3 a_{1} a_{0}^{2}=0, \\
& a_{1} b_{0} b_{1} k_{1}^{2} \log ^{2}(a)-a_{0} b_{1}^{2} k_{1}^{2} \log ^{2}(a)-a_{1} b_{0} b_{1} k_{2} \log (a) \\
& +a_{0} b_{1}^{2} k_{2} \log (a)-2 a_{1} b_{0} b_{1}-a_{0} b_{1}^{2}+3 a_{0} a_{1}^{2}=0,
\end{aligned}
$$


which its solution yields the following new explicit exact solutions to (1).

$$
\begin{aligned}
& a_{0}=0, a_{1}=\mp b_{1}, b_{0}=b_{0}, b_{1}=b_{1} \text {, } \\
& k_{1}=-\frac{1}{\sqrt{2} \log (a)}, k_{2}=-\frac{3}{2 \log (a)} \text {. } \\
& a_{0}=0, a_{1}=\mp b_{1}, b_{0}=b_{0}, b_{1}=b_{1} \text {, } \\
& k_{1}=\frac{1}{\sqrt{2} \log (a)}, k_{2}=-\frac{3}{2 \log (a)} \text {. } \\
& a_{0}=\mp b_{0}, a_{1}=0, b_{0}=b_{0}, b_{1}=b_{1} \text {, } \\
& k_{1}=-\frac{1}{\sqrt{2} \log (a)}, k_{2}=\frac{3}{2 \log (a)} \text {. } \\
& a_{0}=\mp b_{0}, a_{1}=0, b_{0}=b_{0}, b_{1}=b_{1} \text {, } \\
& k_{1}=\frac{1}{\sqrt{2} \log (a)}, k_{2}=\frac{3}{2 \log (a)} \text {. } \\
& u_{1,2}(x, t)=\frac{\mp b_{1} a^{-\left(\frac{1}{\sqrt{2} \log (a)} x+\frac{3}{2 \log (a)} \frac{t y}{y}\right)}}{b_{1} a^{-\left(\frac{1}{\sqrt{2} \log (a)} x+\frac{3}{2 \log (a)} \frac{t y}{y}\right)}+b_{0}}, \quad a \neq 1 \\
& u_{3,4}(x, t)=\frac{\mp b_{1} a^{\left(\frac{1}{\sqrt{2} \log (a)} x-\frac{3}{2 \log (a)} \frac{y}{y}\right)}}{b_{1} a^{\left(\frac{1}{\sqrt{2} \log (a)} x-\frac{3}{2 \log (a)} \frac{y}{y}\right)}+b_{0}}, \quad a \neq 1 \\
& u_{5,6}(x, t)=\frac{\mp b_{0}}{b_{1} a^{\left(-\frac{1}{\sqrt{2} \log (a)} x+\frac{3}{2 \log (a)} \frac{t^{y}}{y}\right)}+b_{0}}, \quad a \neq 1 \\
& u_{7,8}(x, t)=\frac{\mp b_{0}}{b_{1} a^{\left(\frac{1}{\sqrt{2} \log (a)} x+\frac{3}{2 \log (a)} \frac{y}{y}\right)}+b_{0}}, \quad a \neq 1
\end{aligned}
$$

We now again consider (9) to solve by utilizing the hyperbolic function approach.

Case1: $\frac{d \rho}{d \xi}=\sinh (\rho)$ Through homogenous balancing principle, the terms $U^{\prime \prime}$ and $U^{3}$ gives $N=1$ and the non-trivial solution (8) becomes

$$
U(\eta)=B_{1} \sinh (\rho)+A_{1} \cosh (\rho)+A_{0} .
$$

By setting the above non-trivial solution (19) in (9) and equating the coefficients to zero in the resultant equation, we reach a set of nonlinear polynomial equations.

$$
\begin{aligned}
& A_{0}^{3}+3 A_{1}^{2} A_{0}-A_{0}=0, \\
& 3 A_{1} B_{1}^{2}-2 A_{1} k_{1}^{2}+A_{1}^{3}=0, \\
&-3 A_{1} B_{1}^{2}+2 A_{1} k_{1}^{2}+3 A_{1} A_{0}^{2}-A_{1}=0, 0 \\
& 3 A_{1}^{2} B_{1}-2 B_{1} k_{1}^{2}+B_{1}^{3}=0, 0 \\
& 3 A_{0}^{2} B_{1}+3 A_{1}^{2} B_{1}-B_{1} k_{1}^{2}-B_{1}=0, 0, \\
& 3 A_{0} B_{1}^{2}+A_{1} k_{2}+3 A_{0} A_{1}^{2}=0, \\
& 6 A_{0} A_{1} B_{1}+B_{1} k_{2}=0 .
\end{aligned}
$$

On solving the obtained set of equations yields the following sets of solutions which will give us the new exact solutions of (1).

$$
\begin{aligned}
& A_{0}=-\frac{1}{2}, A_{1}=-\frac{1}{2}, B_{1}=-\frac{1}{2}, k_{1}=\mp \frac{1}{\sqrt{2}}, k_{2}=-\frac{3}{2}, \\
& A_{0}=-\frac{1}{2}, A_{1}=-\frac{1}{2}, B_{1}=\frac{1}{2}, k_{1}=\mp \frac{1}{\sqrt{2}}, k_{2}=-\frac{3}{2}, \\
& A_{0}=\frac{1}{2}, A_{1}=-\frac{1}{2}, B_{1}=-\frac{1}{2}, k_{1}=\mp \frac{1}{\sqrt{2}}, k_{2}=\frac{3}{2}, \\
& A_{0}=\frac{1}{2}, A_{1}=-\frac{1}{2}, B_{1}=\frac{1}{2}, k_{1}=\mp \frac{1}{\sqrt{2}}, k_{2}=\frac{3}{2} \\
& A_{0}=-\frac{1}{2}, A_{1}=-\frac{1}{2}, B_{1}=0, k_{1}=\mp \frac{1}{2 \sqrt{2}}, k_{2}=-\frac{3}{4}, \\
& A_{0}=\frac{1}{2}, A_{1}=-\frac{1}{2}, B_{1}=0, k_{1}=\mp \frac{1}{2 \sqrt{2}}, k_{2}=\frac{3}{4}, \\
& A_{0}=-\frac{1}{2}, A_{1}=\frac{1}{2}, B_{1}=\frac{1}{2}, k_{1}=\mp \frac{1}{\sqrt{2}}, k_{2}=\frac{3}{2}, \\
& A_{0}=-\frac{1}{2}, A_{1}=\frac{1}{2}, B_{1}=-\frac{1}{2}, k_{1}=\mp \frac{1}{\sqrt{2}}, k_{2}=\frac{3}{2}, \\
& A_{0}=\frac{1}{2}, A_{1}=\frac{1}{2}, B_{1}=0, k_{1}=\mp \frac{1}{2 \sqrt{2}}, k_{2}=-\frac{3}{4}
\end{aligned}
$$

$$
A_{0}=\frac{1}{2}, A_{1}=\frac{1}{2}, B_{1}=-\frac{1}{2}, k_{1}=\mp \frac{1}{\sqrt{2}}, k_{2}=-\frac{3}{2},
$$

$$
A_{0}=\frac{1}{2}, A_{1}=\frac{1}{2}, B_{1}=\frac{1}{2}, k_{1}=\mp \frac{1}{\sqrt{2}}, k_{2}=-\frac{3}{2}
$$

We now write some new exact solutions using (20) to (31) as follows. From (19) and (20) we get,

$$
u(x, t)=-\frac{1}{2} \operatorname{coth}\left(\mp \frac{1}{\sqrt{2}} x+\frac{3}{2} \frac{t^{y}}{y}\right)-\frac{1}{2}-\frac{1}{2} \operatorname{csch}\left(\mp \frac{1}{\sqrt{2}} x+\frac{3}{2} \frac{t^{y}}{y}\right) .
$$

Now from Eqs. (19) and (24) we get,

$$
u(x, t)=-\frac{1}{2} \operatorname{coth}\left(\mp \frac{1}{2 \sqrt{2}} x+\frac{3}{4} \frac{t^{y}}{y}\right)-\frac{1}{2} .
$$


Now from Eqs. (19) and (28) we get,

$u(x, t)=\frac{1}{2} \operatorname{coth}\left(\mp \frac{1}{\sqrt{2}} x-\frac{3}{2} \frac{t^{y}}{y}\right)-\frac{1}{2}-\frac{1}{2} \operatorname{csch}\left(\mp \frac{1}{\sqrt{2}} x-\frac{3}{2} \frac{t^{y}}{y}\right)$.

The other solutions can be formulated on the similar way. Case2: $\frac{d \rho}{d \xi}=\cosh (\rho)$ and for $N=1$, the non-trivial solution (8) becomes

$$
U(\eta)=A_{0}+B_{1} \cot (\rho)-A_{1} \csc (\rho) .
$$

By setting the above non-trivial solution in (9) and equating the coefficients to zero in the resultant equation, we reach the following set of polynomial equations.

$$
\begin{aligned}
&-3 A_{0} B_{1}^{2}+A_{0}^{3}-A_{0}=0, \\
& 3 A_{1} B_{1}^{2}-A_{1} k_{1}^{2}-3 A_{1} A_{0}^{2}+A_{1}=0,= \\
&-3 A_{1} B_{1}^{2}+2 A_{1} k_{1}^{2}-A_{1}^{3}=0, \\
& 3 A_{0}^{2} B_{1}+3 A_{1}^{2} B_{1}-2 B_{1} k_{1}^{2}-B_{1}=0, \\
& 3 A_{1}^{2} B_{1}-2 B_{1} k_{1}^{2}+B_{1}^{3}=0, \\
&-6 A_{0} A_{1} B_{1}-A_{1} k_{2}=0, \\
& 3 A_{0} B_{1}^{2}+3 A_{0} A_{1}^{2}+B_{1} k_{2}=0 .
\end{aligned}
$$

On solving this system of equations, we obtain the following sets of solutions that will give us the new exact solutions of (1)

$$
\begin{gathered}
A_{0}=-\frac{1}{2}, A_{1}=-\frac{i}{2}, B_{1}=-\frac{i}{2}, k_{1}=\mp \frac{i}{\sqrt{2}}, k_{2}=-\frac{3 i}{2}, \\
A_{0}=-\frac{1}{2}, A_{1}=\frac{i}{2}, B_{1}=-\frac{i}{2}, k_{1}=\mp \frac{i}{\sqrt{2}}, k_{2}=-\frac{3 i}{2}, \\
A_{0}=\frac{1}{2}, A_{1}=-\frac{i}{2}, B_{1}=-\frac{i}{2}, k_{1}=\mp \frac{i}{\sqrt{2}}, k_{2}=\frac{3 i}{2}, \\
A_{0}=\frac{1}{2}, A_{1}=\frac{i}{2}, B_{1}=-\frac{i}{2}, k_{1}=\mp \frac{i}{\sqrt{2}}, k_{2}=\frac{3 i}{2} \\
A_{0}=-\frac{1}{2}, A_{1}=0, B_{1}=-\frac{i}{2}, k_{1}=\mp \frac{i}{2 \sqrt{2}}, k_{2}=-\frac{3 i}{4}, \\
A_{0}=\frac{1}{2}, A_{1}=0, B_{1}=-\frac{i}{2}, k_{1}=\mp \frac{i}{2 \sqrt{2}}, k_{2}=\frac{3 i}{4} \\
A_{0}=-\frac{1}{2}, A_{1}=0, B_{1}=\frac{i}{2}, k_{1}=\mp \frac{i}{2 \sqrt{2}}, k_{2}=\frac{3 i}{4}, \\
A_{0}=\frac{1}{2}, A_{1}=0, B_{1}=\frac{i}{2}, k_{1}=\mp \frac{i}{2 \sqrt{2}}, k_{2}=-\frac{3 i}{4}
\end{gathered}
$$
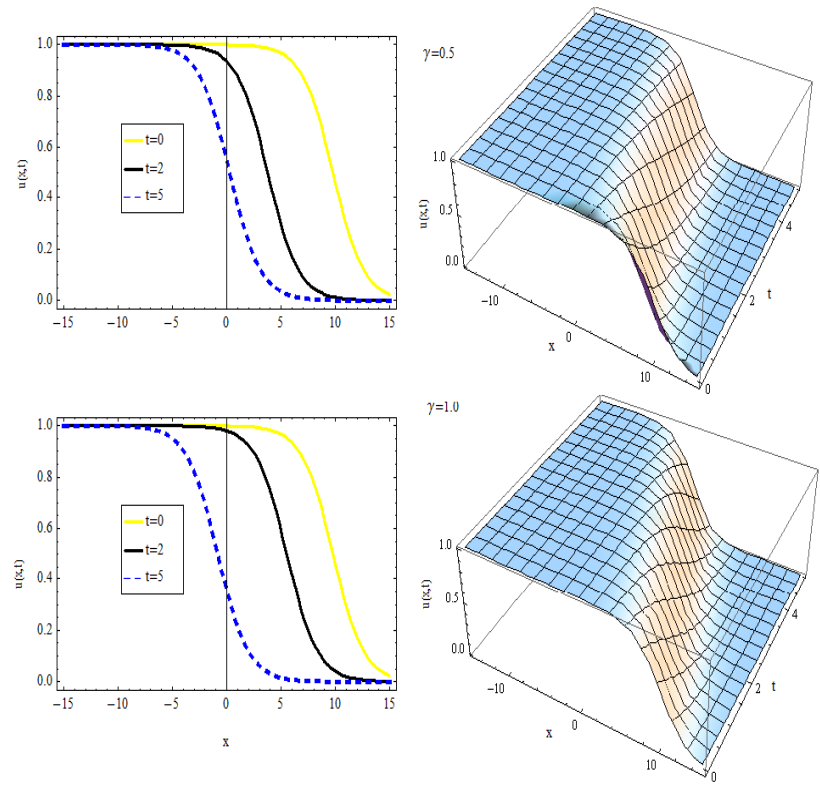

Figure 1: Graphs of (11) for conformable time-fractional Cahn-Allen equation.

$$
\begin{aligned}
& A_{0}=-\frac{1}{2}, A_{1}=-\frac{i}{2}, B_{1}=\frac{i}{2}, k_{1}=\mp \frac{i}{\sqrt{2}}, k_{2}=\frac{3 i}{2}, \\
& A_{0}=-\frac{1}{2}, A_{1}=\frac{i}{2}, B_{1}=\frac{i}{2}, k_{1}=\mp \frac{i}{\sqrt{2}}, k_{2}=\frac{3 i}{2}, \\
& A_{0}=\frac{1}{2}, A_{1}=-\frac{i}{2}, B_{1}=\frac{i}{2}, k_{1}=\mp \frac{i}{\sqrt{2}}, k_{2}=-\frac{3 i}{2}, \\
& A_{0}=\frac{1}{2}, A_{1}=\frac{i}{2}, B_{1}=\frac{i}{2}, k_{1}=\mp \frac{i}{\sqrt{2}}, k_{2}=-\frac{3 i}{2} .
\end{aligned}
$$

We now write some new complex trigonometric solutions using (36) to (47) as follows. From (36) and Case-2 of (19) we get,

$u(x, t)=-\frac{i}{2} \cot \left(\mp \frac{i}{\sqrt{2}} x+\frac{3 i}{2} \frac{t^{y}}{y}\right)-\frac{1}{2}+\frac{i}{2} \csc \left(\mp \frac{i}{\sqrt{2}} x+\frac{3 i}{2} \frac{t^{y}}{y}\right)$.

From (40) and Case-2 of (19), we obtain

$$
u(x, t)=-\frac{i}{2} \cot \left(\mp \frac{i}{2 \sqrt{2}} x+\frac{3 i}{4} \frac{t^{y}}{y}\right)-\frac{1}{2} .
$$

From (44) and Case-2 of (19), we procure

$$
u(x, t)=\frac{i}{2} \cot \left(\mp \frac{i}{\sqrt{2}} x-\frac{3 i}{2} \frac{t^{y}}{y}\right)-\frac{1}{2}+\frac{i}{2} \csc \left(\mp \frac{i}{\sqrt{2}} x-\frac{3 i}{2} \frac{t^{y}}{y}\right) .
$$

The other solutions can be formulated on the same way. 


\section{Some graphical illustrations}

In this section, we give some graphical illustrations of the acquired solutions of our equations. The 2-dimensional and 3-dimensional plots of certain solutions are presented as follows.
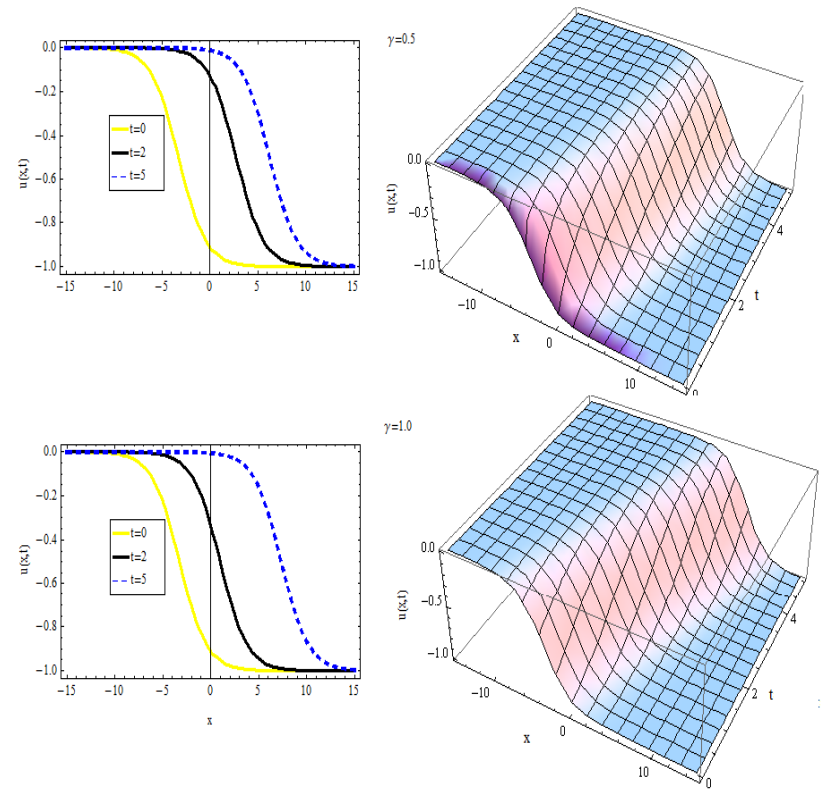

Figure 2: Graphs of (12) for conformable time-fractional Cahn-Allen equation.
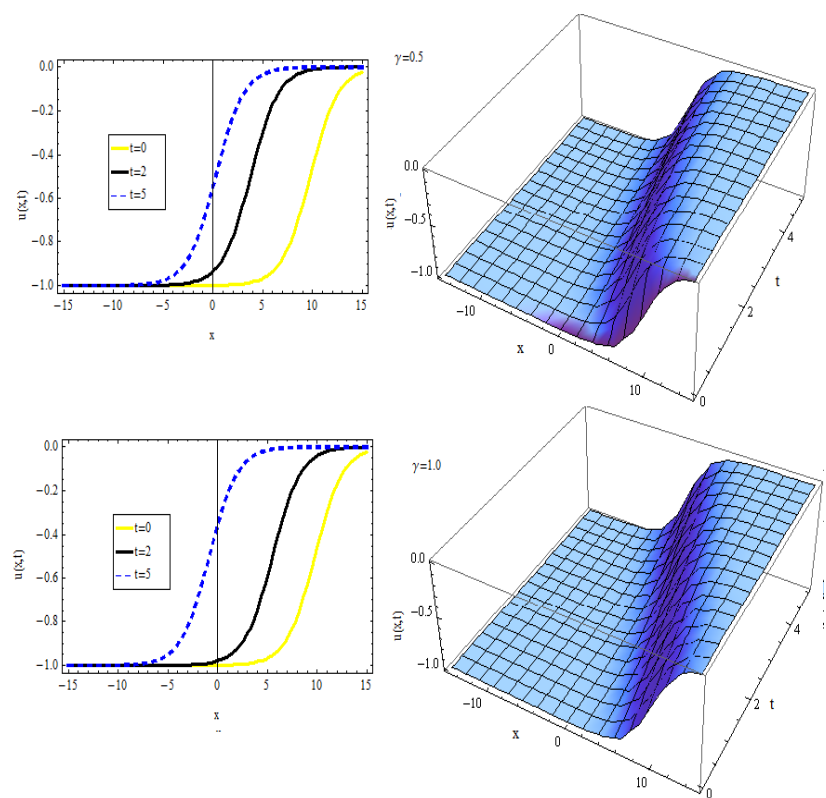

Figure 3: Graphs of (13) for conformable time-fractional Cahn-Allen equation.
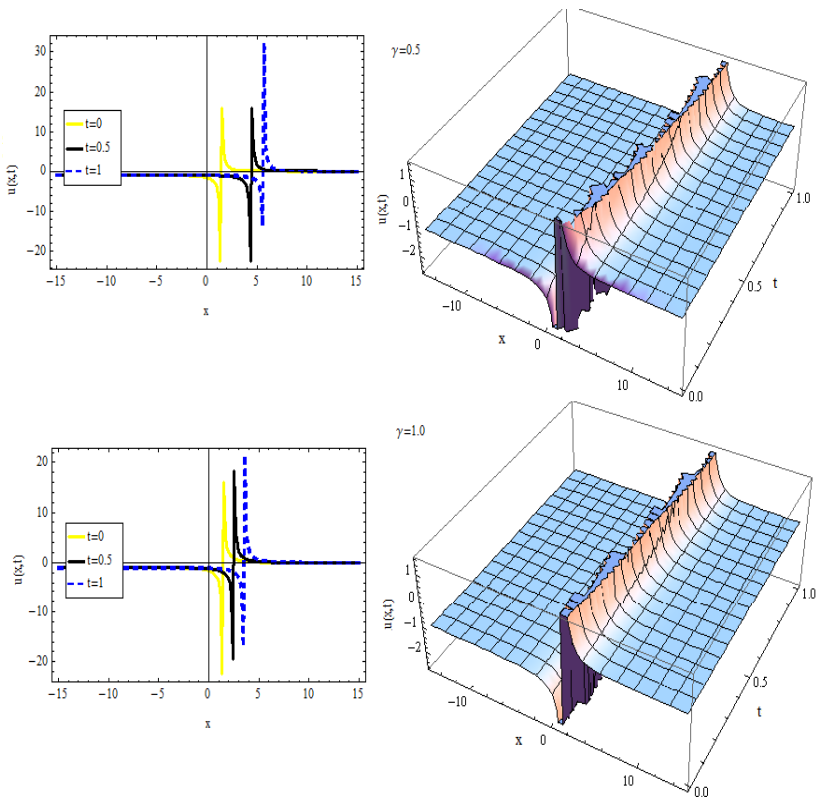

Figure 4: Graph of (32) for conformable time-fractional Cahn-Allen equation.
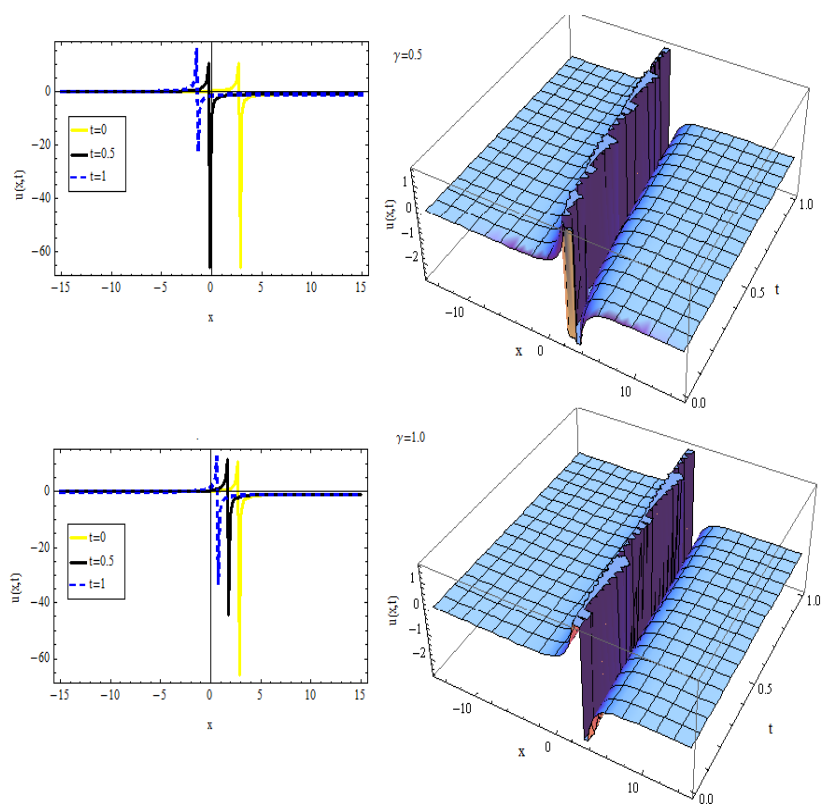

Figure 5: Graphs of (33) for conformable time-fractional Cahn-Allen equation. 

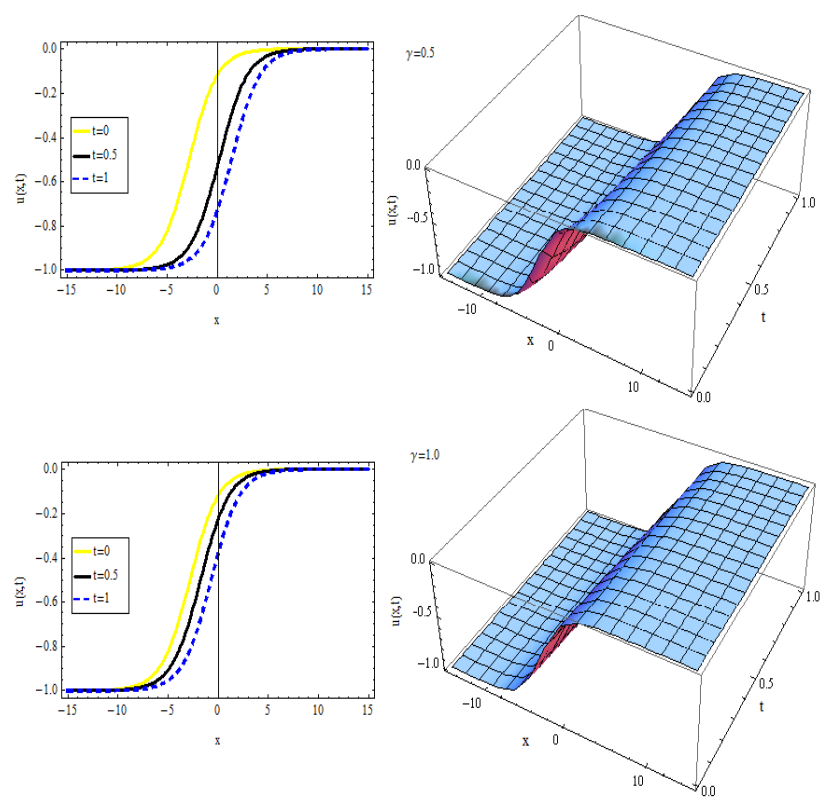

Figure 6: Graphs of (34) for conformable time-fractional Cahn-Allen equation.
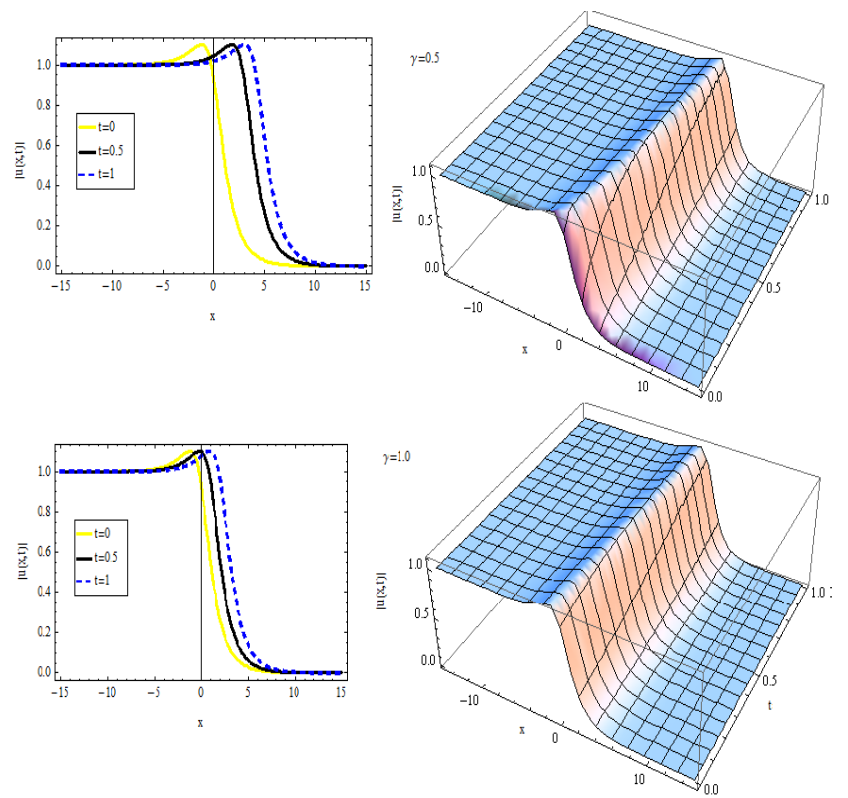

Figure 7: Graphs of (48) for conformable time-fractional Cahn-Allen equation.
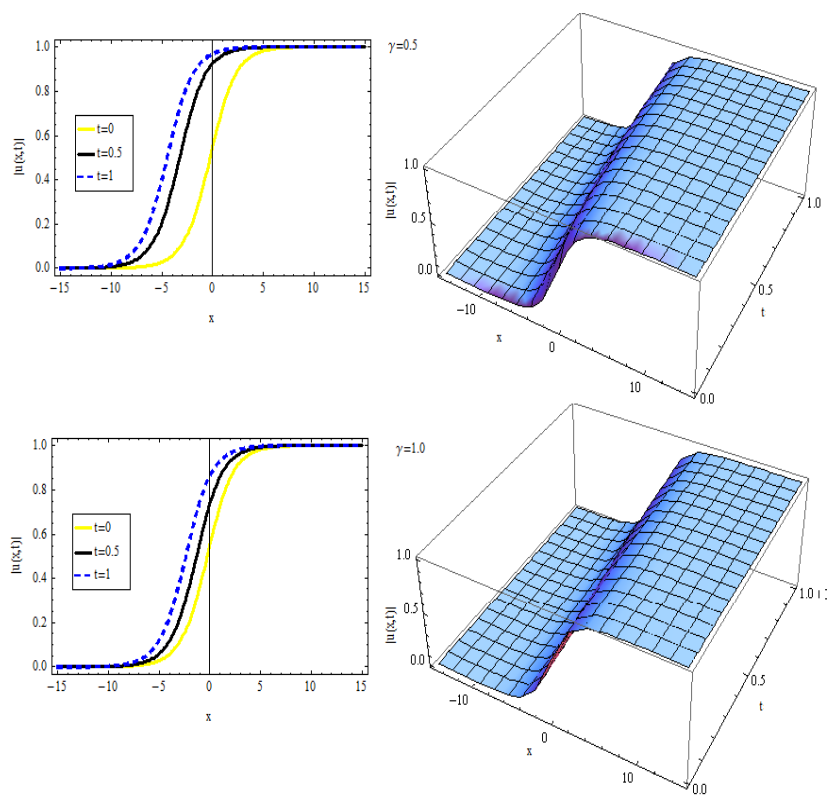

Figure 8: Graphs of (49) for conformable time-fractional Cahn-Allen equation.
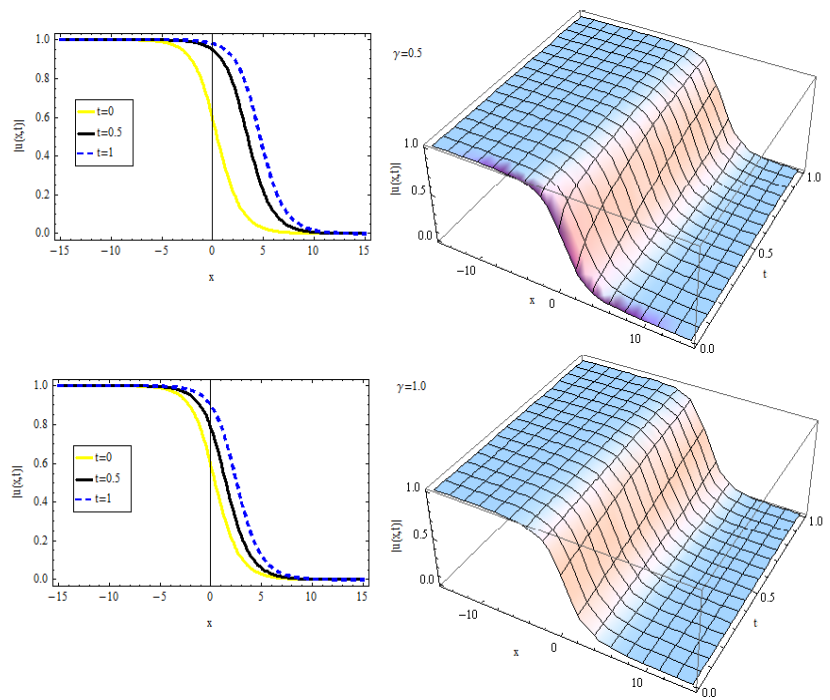

Figure 9: Graphs of (50) for conformable time-fractional Cahn-Allen equation. 


\section{Conclusion}

In this paper an efficient method was built up to solve conformable time-fractional Cahn-Allen equation. This idea is based on the idea of hyperbolic function and expa function, which is a known method for solving diffusion equations. These methods are very powerful with minimum algebraic work. The computations are done using Maple 18.

\section{References}

[1] Korkmaz A., Hepson O.E., Hosseini K., Rezazadeh H., Eslami M., Sine-gordon expansion method for exact solutions to conformable time fractional equations in RLW-class, J. of King Saud Univ. Sci., 2018, 32(1), 567-574.

[2] Rezazadeh H., Tariq H., Eslami M., Mirzazadeh M., Zhou Q., New exact solutions of nonlinear conformable time-fractional phi-4 equation. Chin. J. of Phy., 2018, 56(6), 2805-2816.

[3] Osman M.S., Alper Korkmaz, Rezazadeh H., Mirzazadeh M., Eslami N., Zhou Q. The unifed method for conformable time fractional schrödinger equation with perturbation terms. Chin. J. of Phy., 2018, 56(5), 2500-2506.

[4] Rezazadeh H., Korkmaz A., Eslami M., Vahidi J., Asghari R., Traveling wave solution of conformable fractional generalized reaction duffing model by generalized projective riccati equation method, Opti. and Q. Elect., 2018, 50(3), 150.

[5] Raslan K.R., El-Danaf T.S., Ali K.K., Exact solution of spacetime fractional coupled Ew and coupled MEW equations using modified kudryashov method, Commu. in Theor. Phy., 2017, 68(1), 49.

[6] Raslan K.R., El-Danaf T.S., Ali K.K., Exact solution of the spacetime fractional coupled EW and coupled MEW equations, The Eur. Phy. J. Plus, 2017, 132(7), 319.

[7] Karaagac B., New exact solutions for some fractional order dierential equations via improved sub-equation method. Disc. and Contin. Dy. Sys.-S, 2018, 12(3), 447.

[8] Ucar Y., Yagmurlu N.M., Tasbozan O., Esen A., Numerical solution of some fractional partial differential equations using collocation finite element method, Progr. Fract. Diff. Appl., 2015, 1, 157-164.
[9] Bekir A., Multisoliton solutions to cahn-allen equation using double exp-function method. Physics of Wave Phenomena, 2012, 20(2), 118-121.

[10] Filiz T., Bekir A., Travelling wave solutions of the cahn-allen equation by using

first integral method, Appl. Math. and Comput., 2009, 207(1), 279-282.

[11] Ozkan G., Bekir A., Cevikel A.C., A variety of exact solutions for the time fractional cahn-allen equation, The Eur. Phy. J. Plus, 2015, 130(7), 146.

[12] Tariq H., Ghazala A., New approach for exact solutions of time fractional cahn-allen equation and time fractional phi-4 equation. Phy. A: Stat. Me-chan. and its Appli., 2017, 473, 352-362.

[13] Ali A.T., Hassan E.R., General expa function method for nonlinear evolution equations, Appl. Math. Comput., 2010, 217, 451-459.

[14] Hosseini K., Ayati Z., Ansari R., New exact solution of the tzitzeica type equations in nonlinear optics using the expa function method, J. Mod. Opt., 2018, 65(7), 847-851.

[15] Zayed E.M.E., Al-Nowehy A.G., Generalized kudryashov method and general exp a function method for solving a high order nonlinear schrödinger equation, J. S. Explor., 2017, 6, 1-26.

[16] Xie F., Yan Z., Zhang H., Explicit and exact traveling wave solutions of whitham-broer-kaup shallow water equations, Phy. Lett. A, 2001, 285(1), 76-80.

[17] Bai C., Exact solutions for nonlinear partial differential equation: a new approach, Phy. Lett. A, 2001, 288(3):191-195.

[18] Hosseini K., Zabihi A., Samadani F., Ansari R., New explicit exact solutions of the unstable nonlinear schrödinger's equation using the expa and hyperbolic function methods, Opti. and Q. Electr., 2018, 50(2), 82.

[19] Khalil R., Al Horani M., Yousef A., Sababheh M., A new defination of fractional derivative, J. Comput. Appl. Math., 2014, 264, 65-70.

[20] Zafar A., Rational exponential solutions of conformable spacetime fractional equal width equations, Non. Eng., 2019, 8, 350-355

[21] Seadawy A.R., Kumar D., Hosseini K., Samadani F., The system of equations for the ion sound and langmuir waves and its new exact solutions, Resu. in Phy., 2018, 9, 1631-1634. 